\title{
PI3K $\beta$ downstream of GPCRs - crucial partners in oncogenesis
}

\section{Hashem A. Dbouk, Oscar Vadas, Roger L. Williams, and Jonathan M. Backer}

Of the class IA PI3Ks, p110 $\beta$ is the only member to simultaneously signal downstream of both Receptor Tyrosine Kinases (RTKs) and G Protein-Coupled Receptors (GPCRs). While the mechanism of RTKmediated activation of p85/p110 heterodimers has been characterized $[1,2]$, defining the mechanism of G $\beta \gamma$ mediated activation of p110 $\beta$ downstream of GPCRs has been challenging due to the transient nature of the interaction. The binding of $\mathrm{p} 110 \beta$ to $\mathrm{G} \beta \gamma$ is weak, but is reinforced by interactions with lipid membranes, where G $\beta \gamma$ resides. A combination of sequence analysis and hydrogen/deuterium exchange (HDX) coupled to mass spectrometry (MS) experiments has enabled us to map the regions of interaction on both p110 $\beta$ and G $\beta \gamma$ [3]. Furthermore, HDX-MS data analysis provided a unique insight into the mechanism of $\mathrm{p} 110 \beta$ activation by $\mathrm{G} \beta \gamma$, showing that $\mathrm{p} 110 \beta$ kinase domain makes stronger interaction with membranes in the presence of G $\beta \gamma$. The regulatory $\mathrm{p} 85$ subunit inhibits basal activity of $\mathrm{p} 110 \beta$, largely by the inhibitory contacts that the $\mathrm{nSH} 2$ and the $\mathrm{cSH} 2$ domains of $\mathrm{p} 85$ make with the $\mathrm{p} 110 \beta$ subunit $[1$, 2]. The $\mathrm{nSH} 2$ contacts the helical domain of $\mathrm{p} 110 \beta$ close to the residues essential for $G \beta \gamma$ binding. Whether the $\mathrm{nSH} 2$ has to disengage from $\mathrm{p} 110 \beta$ to allow $\mathrm{G} \beta \gamma$ binding is still uncertain. In vitro, p110 $\beta / p 85$ truncation constructs lacking either the $\mathrm{nSH} 2$ or both $\mathrm{SH} 2$ domains are still activated by $\mathrm{G} \gamma$. In contrast, activation of $\mathrm{p} 110 \beta / \mathrm{p} 85$ constructs by tyrosyl phosphorylated phosphopeptides require the presence of at least one p85 $\mathrm{SH} 2$ domain. Thus, G $\beta \gamma$ and phosphopeptides activate the enzyme via distinct mechanisms, resulting in synergistic activation when both activators are present. Whether there is an allosteric component of activation in addition to membrane recruitment mediated by G $\beta \gamma$ remains an open question. Based on the current results and on the structure of a membrane interacting heterotrimeric G-protein, we can begin to model how the activated p110 $\beta$ would interact with membranes. In this model, G $\beta \gamma$ makes no direct contact with the kinase domain of p110 $\beta$, suggesting a mechanism where $G \beta \gamma$ stimulates p110 $\beta$ activity by increasing its membrane residence time. A similar mechanism has been proposed for the other G $\beta \gamma$-sensitive PI3K, p110 $\gamma$ [4]. The crystal structure of p110 $\beta$ in a complex with G $\beta \gamma$ will answer many remaining questions.

Wild-type $\mathrm{p} 110 \beta$ is transforming when overexpressed in fibroblasts [5], and it is the major p110 isoform required for driving $\mathrm{PTEN}^{-/}$tumors [6]. However, it has not been previously possible to determine whether GPCR activation of $\mathrm{p} 110 \beta / \mathrm{p} 85$ was required in these cases. Using a mutant $\mathrm{p} 110 \beta$ or a cell-permeable peptide,

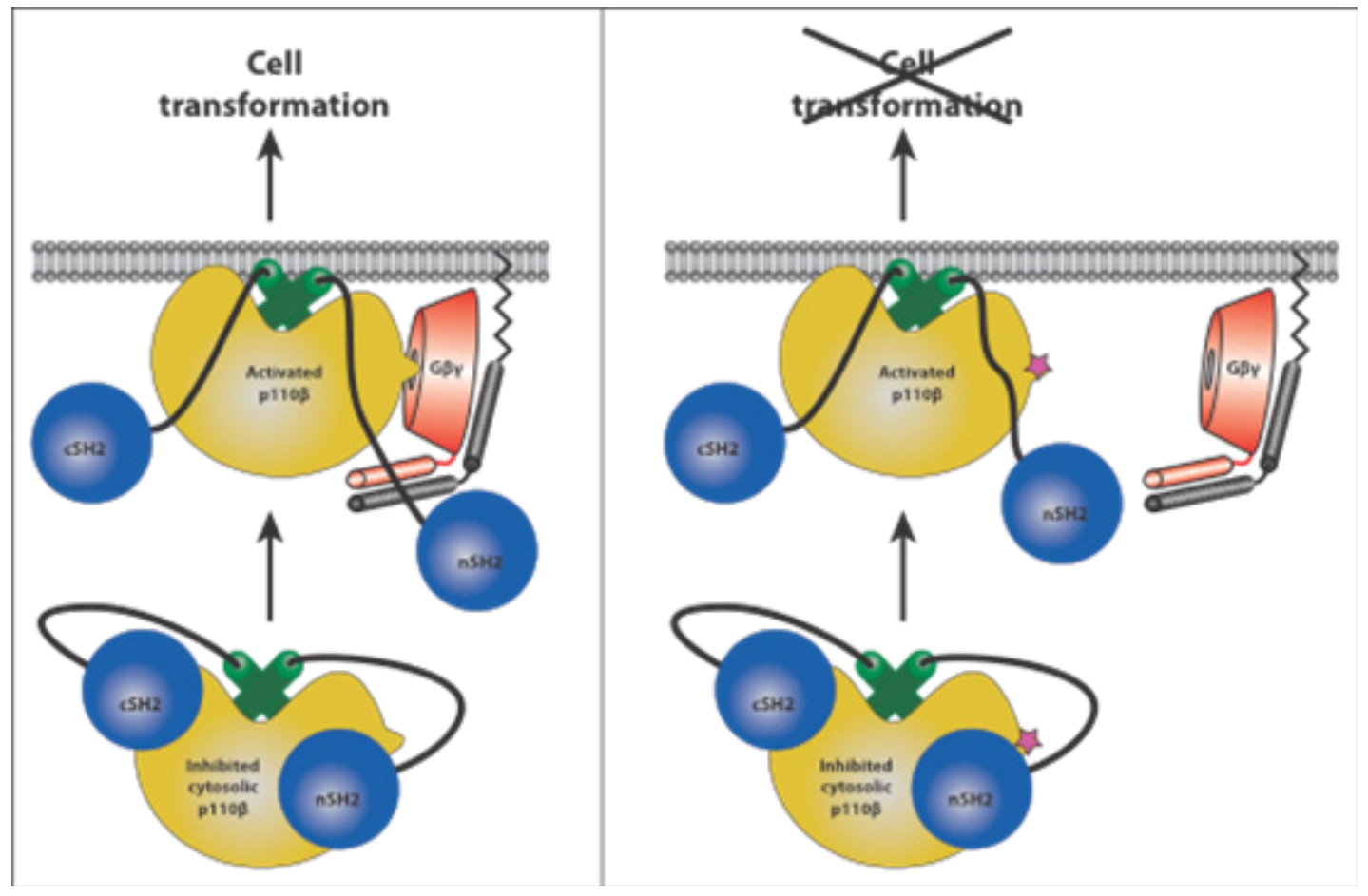


both of which block GPCR- mediated but not RTKmediated activation of $\mathrm{p} 110 \beta / \mathrm{p} 85$, we showed that GPCR inputs to $\mathrm{p} 110 \beta / \mathrm{p} 85$ are required for transformation, proliferation, chemotaxis, and invasion driven by either p110 $\beta$ over-expression or stimulation with GPCR ligands [3]. Furthermore, a requirement for GPCR activation of p110ß/p85 was seen in the growth of PTEN ${ }^{-/}$cell lines, but not PTEN ${ }^{+/+}$cells. Surprisingly, the peptide inhibitor of $\mathrm{p} 110 \beta / \mathrm{G} \beta \gamma$ binding blocked the growth of $\mathrm{PTEN}^{-/}$cells, whereas the p110ß-specific kinase inhibitor, TGX-221, did not. This suggests a role for a $\mathrm{G} \beta \gamma$-mediated scaffolding function of $\mathrm{p} 110 \beta$ in proliferation. This is consistent with previous studies showing that some functions of $\mathrm{p} 110 \beta$ are kinase independent $[7,8]$. Our work suggests that in some tumors, inhibitors specifically targeting the G $\beta \gamma$ p110 $\beta$ interaction might be more potent than inhibitors targeting $\mathrm{p} 110 \beta$ catalytic activity. Our study also suggests that the identification of the GPCRs that drive PTEN ${ }^{-1-}$ tumors could provide an important alternative therapeutic approach for the treatment of these tumors. Understanding the regulation of $p 110 \beta$ catalytic activity, as well as defining its scaffolding functions, will be important in developing drugs that target its functions in human disease.

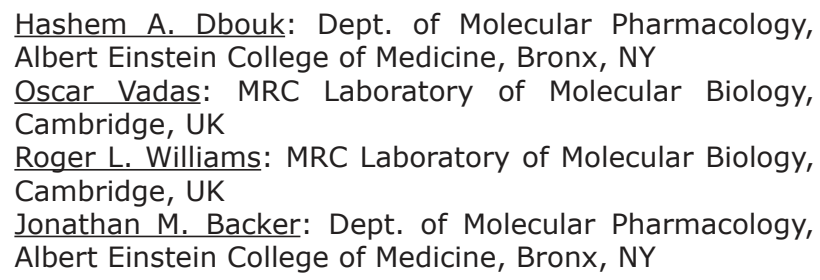

Correspondence: Roger L. Williams, email rlw@mrc-Imb. cam.ac.uk

Correspondence: Jonathan M. Backer, email jonathan. backer@einstein.yu.edu

Received: December 14, 2012;

Published: December 18, 2012;

\section{REFERENCES}

1. Vadas O, Burke JE, Zhang X, Berndt A and Williams RL. Sci Signal. 2011; 4(195):re2.

2. Backer JM. Curr Top Microbiol Immunol. 2010; 346:87114.

3. Dbouk HA et al. Science signaling. 2012; 5(253):ra89.

4. Brock C et al. J Cell Biol. 2003; 160(1):89-99.

5. Kang S et al. Proc Natl Acad Sci U S A. 2006; 103(5):12891294.

6. Wee $\mathrm{S}$ et al. Proc Natl Acad Sci U S A. 2008; 105(35):13057-13062.

7. Ciraolo E et al. Science signaling. 2008; 1(36):ra3.

8. Jia S et al. Nature. 2008; 454(7205):776-779. 